\title{
Stearoyl CoA desaturase 1 is elevated in obesity but protects against fatty acid-induced skeletal muscle insulin resistance in vitro
}

\author{
S. K. Pinnamaneni • R. J. Southgate • M. A. Febbraio • \\ M. J. Watt
}

Received: 29 June 2006 / Accepted: 17 July 2006 / Published online: 11 October 2006

(C) Springer-Verlag 2006

\begin{abstract}
Aims/hypothesis Stearoyl CoA desaturase 1 (SCD1) is implicated in mediating obesity and insulin resistance. Paradoxically, SCD1 converts saturated fatty acids, the lipid species implicated in mediating insulin resistance, to monounsaturated fatty acids. The aim of the present study was to assess the molecular mechanisms that implicate SCD1 in the aetiology of fatty acid-induced insulin resistance.

Methods SCD1 protein was transiently decreased or increased in rat L6 skeletal muscle myotubes using SCD1 short interfering RNA (siRNA) or liposome-mediated transfection of pcDNA3.1/Hygro-mSCD1, respectively.

Results Reducing SCD1 protein resulted in marked esterification of exogenous fatty acids into diacylglycerol (DAG) and ceramide. Insulin-stimulated Akt activity and phosphorylation and 2-deoxyglucose uptake were reduced with SCD1 siRNA. Exposure of L6 myotubes to palmitate abolished insulin-stimulated glucose uptake in both control and SCD1 siRNA myotubes. Overexpression of SCD1 resulted in triacylglycerol esterification but attenuated ceramide and DAG accumulation and protected myotubes from fatty acid-induced insulin resistance.
\end{abstract}

Electronic supplementary material Supplementary material is available for this article at http://dx.doi.org/10.1007/s00125-006$0427-9$ and is accessible to authorised users.

S. K. Pinnamaneni $\cdot$ R. J. Southgate $\cdot$ M. A. Febbraio $\cdot$ M. J. Watt Cellular and Molecular Metabolism Laboratory,

School of Medical Sciences, RMIT University,

Melbourne, Victoria, Australia

M. J. Watt $(\bowtie)$

St Vincent's Institute of Medical Research and Department of Medicine, University of Melbourne,

Fitzroy, Victoria, Australia

e-mail: mwatt@svi.edu.au
Conclusions/interpretation SCD1 protects from cellular toxicity in L6 myotubes by preventing excessive accumulation of bioactive lipid metabolites.

Keywords Ceramide $\cdot$ Diacylglycerol $\cdot$ Insulin resistance . Obesity Palmitate $\cdot$ Skeletal muscle .

Stearoyl CoA desaturase

$\begin{array}{ll}\text { Abbreviations } \\ \text { 2DG } & \text { 2-deoxyglucose } \\ \text { DAG } & \text { diacylglycerol } \\ \text { MEM } & \text { minimal essential medium } \\ \text { SCD1 } & \text { stearoyl CoA desaturase 1 } \\ \text { siRNA } & \text { short interfering RNA }\end{array}$

\section{Introduction}

Skeletal muscle insulin resistance is a central feature of obesity and type 2 diabetes [1] and aberrant fatty acid metabolism is an underlying feature of these conditions. Lipid oversupply to non-adipose tissues associated with glucose homeostasis, such as skeletal muscle, liver and the pancreas, leads to tissue dysfunction. For example, skeletal muscle triacylglycerol content is positively associated with insulin resistance in humans and rodents [2, 3] and situations in which fatty acids are elevated, such as chronic high-fat feeding [4-6] and transient infusion of triglyceride and heparin [7-10], induce insulin resistance. Studies conducted in skeletal muscle myotube cultures, a model that negates the influence of extraneous factors, such as sympathoadrenal activation, hormonal fluxes and crosstalk between tissues, show that saturated fatty acids inhibit insulin signalling and deoxyglucose uptake [7-13] via 
accumulation of fatty acyl CoA metabolites and activation of several serine/threonine kinases (inhibitor of $\kappa \mathrm{B}$ kinase, c-jun terminal amino kinase) known to impair insulin signal transduction $[10,14,15]$.

Stearoyl CoA desaturase 1 (SCD1) is an endoplasmic reticulum-bound enzyme that converts saturated fatty acids (palmitoyl and stearoyl-CoA) to monounsaturated fatty acids [16], and may be a critical protein in the regulation of insulin action in skeletal muscle. The quantity and distribution of saturated and unsaturated fatty acids affects many cellular processes, including phospholipid composition and membrane fluidity, the generation of second messengers in signalling cascades, metabolic fuel storage in the form of triacylglycerols, cellular differentiation, and apoptosis [17-20]. With reference to insulin resistance, recent studies have indicated that exposure of skeletal muscle to excess saturated fatty acid results in the generation of the fatty acyl-CoA metabolites diacylglycerol (DAG) and ceramide, which interfere directly with insulin signal transduction [7-13]. Thus, inhibiting SCD1 function would be expected to result in the accumulation of fatty acid metabolites that are deleterious to insulin signalling and, accordingly, the development of fatty acid-induced insulin resistance.

Paradoxically, the weight of experimental evidence suggests that SCD1 contributes to the pathogenesis of obesity and insulin resistance. SCD1 content is elevated in the livers of insulin-resistant $o b / o b$ and lipoatrophic mice $[21,22]$ and in animals rendered insulin-resistant by high-fat feeding [23]. Moreover, SCD1-null (abJ/abJ, SCD1-/-) mice $[21,24]$ and mice treated with SCD1specific antisense oligonucleotide inhibitors [25] exhibit a leaner phenotype and resistance to diet-induced obesity [21, 24]. However, the interpretation of SCD1 function in these latter studies is confounded by reports of increased energy expenditure [24, 25] and basal thermogenesis [26], and adaptive alterations in metabolic genes.

Skeletal muscle is an important tissue for fat metabolism [27] and insulin-stimulated glucose disposal [28], and is accordingly a pivotal tissue for energy homeostasis and insulin resistance. The biological role of skeletal muscle SCD1 in these processes remains poorly understood. A recent study indicated that SCD1 was elevated in skeletal muscle of obese individuals and contributed to a decrease in fatty acid oxidation and increased triacylglycerol esterification [29]. It is unlikely that SCD1 contributes to skeletal muscle insulin resistance via this pathway since triacylglycerol does not directly interfere with insulin signal transduction and increased intramuscular triacylglycerol actually correlates with enhanced insulin sensitivity in some models [30]. In a recent study that examined SCD1 overexpression in primary human cell culture [29], lipid metabolites known to affect insulin signalling were not measured, nor was insulin-stimulated glucose uptake or the associated signalling pathways. Accordingly, the aim of the present study was to directly examine the role of SCD1 in fatty acid metabolism and insulin resistance in skeletal muscle culture.

\section{Materials and methods}

\section{Animal experiments}

Eight-week-old male C57B1/6J mice and 4-week-old male Wistar rats (Monash Animal Sciences, Vic., Australia) were fed either a chow (4\% calories from fat) or a high-fat $(53 \%$ fat) diet and were provided with food and water ad libitum for 10 weeks. In addition, 20-week-old male $d b / d b$ mice (a gift from G. Tesch, Monash University, Australia) were also studied. Overnight-fasted animals were anaesthetised by intraperitoneal injection of sodium pentobarbital $(60 \mathrm{mg} / \mathrm{kg}$ body mass) and the gastrocnemius muscle was rapidly dissected out, immediately frozen in liquid nitrogen and stored at $-80^{\circ} \mathrm{C}$ until analyses. Animals were killed by lethal injection of sodium pentobarbital. All experimental procedures were approved by the Royal Melbourne Institute of Technology Animal Ethics Committee and the 'Principles of laboratory animal care' were followed.

\section{Cell culture}

L6 myoblasts were grown in $\alpha$-minimal essential medium (MEM) containing 10\% fetal bovine serum (v/v) and $1 \%$ penicillin/streptomycin ( $\mathrm{vol} / \mathrm{vol})$ in an atmosphere of $95 \%$ $\mathrm{O}_{2} / 5 \% \mathrm{CO}_{2}$ at $37^{\circ} \mathrm{C}$ until they reached $50-60 \%$ confluence. Cells were then exposed to short interfering (si) RNAs or cDNA for 16-20 h (described below). Myoblasts were differentiated into multinucleated myotubes by serum withdrawal (changing the medium to Dulbecco's MEM supplemented with $2 \%$ horse serum and $1 \%$ penicillinstreptomycin $[\mathrm{v} / \mathrm{v}])$. After 3 days, most cells had fused to form multinucleated myotubes and were subjected to experimental procedures. Stock fatty acid solutions were prepared by conjugating $100 \mathrm{mmol} / \mathrm{l}$ palmitate, dissolved in ethanol, to $2 \%(\mathrm{w} / \mathrm{v})$ fatty acid-free bovine serum albumin (BSA). This gave a final ratio of NEFA to BSA of 2.5:1.0. Control media contained $2 \% \mathrm{BSA}(\mathrm{w} / \mathrm{v})$ and no lipid.

\section{siRNA}

Two siRNA sequences of rat SCD1 were tested for their capacity to decrease SCD1 protein levels in L6 myotubes. Gene silencing was achieved with equal efficacy with the following pairs of 21-bp oligonucleotide sequences (accession number NM139192, siRNA ID 58064 and siRNA ID 
57973; Ambion, Austin, TX, USA). The oligonucleotide pairs were annealed and reconstituted in RNase-free water. A negative control siRNA was also purchased to account for off-target effects. For transfection, the cell culture medium was changed to antibiotic-free Dulbecco's MEM and $50 \mathrm{pmol}$ of the respective oligonucleotide sequence was added with Lipofectamine 2000 (Invitrogen, Mount Waverley, VIC, Australia) to give a final concentration of $30 \mathrm{nmol} / \mathrm{l}$. The transfection medium was removed after 16-20 h, and cells were washed with phosphate-buffered saline (PBS) and differentiated as described.

\section{Transient transfections}

Each well of a six-well plate of L6 myoblasts was transfected with $0.83 \mu \mathrm{g}$ of DNA (pcDNA3.1/HygromSCD1 was a kind gift from A. Tall, Department of Medicine, Columbia University, NY, USA) using a liposome-mediated transfection procedure. Vector alone (no insert) was used for control experiments. Cells were transfected using a mixture of DOTAP ( $N$-[1-(2,3-dioleoyloxy)propyl]- $N, N, N$-trimethylammonium methylsulphate) and DOSPER (1,3-di-oleoyloxy-2-(6-carboxy-spermyl)propylamid) in filter-sterilised HEPES-buffered saline (42 mmol/1 HEPES, $274 \mathrm{mmol} / \mathrm{l} \mathrm{NaCl}, 10 \mathrm{mmol} / \mathrm{l} \mathrm{KCl}$, $1.4 \mathrm{mmol} / 1 \mathrm{Na}_{2} \mathrm{HPO}_{4}, 11 \mathrm{mmol} / \mathrm{l}$ dextrose, $\mathrm{pH} \mathrm{7.1)}$ and cDNA. The mixture was added to the cells in $1 \mathrm{ml} \alpha$-MEM supplemented with $10 \%$ fetal bovine serum. The transfection medium was removed after 16-20 h, and cells were washed with PBS and differentiated as described. In preliminary experiments using pEGFP.C1, we observed between 20 and $30 \%$ transfection efficiency.

Analytical techniques

\section{Western blotting}

Western blotting was performed in cell lysates and tissue lysates (see Electronic supplementary material). Primary antibodies for SCD1 were obtained from Santa Cruz Biotechnology (Santa Cruz, CA, USA), anti-IRS1 was obtained from Upstate (Lake Placid, NY, USA), whereas IRS1 Y895, p85, Akt and phospho-Akt (Ser473) were obtained from Cell Signaling Technology (Beverly, MA, USA). All data represent protein phosphorylation divided by the total protein content or, where appropriate, by $\alpha$-actin content (Sigma-Aldrich, St Louis, MI, USA).

\section{Akt activity}

Akt activity was assessed using a commercially available kit (catalogue no. 124007; Calbiochem, San Diego, CA, USA). Briefly, this assay involves immunoprecipitation of
Akt from cell lysates followed by the addition of glycogen synthase kinase (GSK) $3 \alpha$, an Akt substrate. Phosphorylated GSK $3 \alpha$ is detected by immunoblotting using a phospho-specific antibody. The quantified protein reflects Akt activity.

\section{Fatty acid metabolism}

Fatty acid metabolism was performed in fully differentiated cells (see Electronic Supplementary Material). Briefly, fully differentiated cells were serum-starved for $4 \mathrm{~h}$ and, after washing twice with PBS, fresh medium $(2 \mathrm{ml})$, consisting of $\alpha$-MEM, $2 \%$ BSA and $500 \mu \mathrm{mol} / 1$ palmitate, $37 \mathrm{kBq} / \mathrm{ml}$ $\left[9,10-{ }^{3} \mathrm{H}\right]$ palmitate (Amersham Biosciences, Little Chalfont, UK), was added to the cells. The reaction was stopped after $2 \mathrm{~h}$ by removing the medium and washing cells three times with ice-cold PBS. Fatty acid oxidation and esterification were then determined [31].

\section{Muscle lipid analysis}

Diacylglycerol and ceramide were extracted and quantified according to the methods of Preiss et al. [32]. Lipids were extracted from myotubes using chloroform : methanol : PBS $+0.2 \%$ SDS (1:2:0.8). Diacylglycerol kinase and ${ }^{32}$ P-labelled ATP $(0.55 \mathrm{GBq} / \mathrm{mmol}$ cold ATP $)$ were added to lysates preincubated with cardiolipin/octylglucoside, and the reaction was stopped after $2 \mathrm{~h}$ by the addition of chloroform: methanol (2:1). Samples were spotted onto thin-layer chromatography plates and developed. ${ }^{32} \mathrm{P}-\mathrm{la}-$ belled phosphatidic acid and ceramide-1-phosphate ceramide were identified, dried, scraped from the thin-layer chromatography plate and counted in a liquid scintillation analyser.

\section{Glucose uptake}

For the determination of 2-deoxy-D- $\left[{ }^{14} \mathrm{C}\right]$ glucose uptake, cells were grown as described and incubated in $0.5 \mathrm{mmol} / 1$ fatty acids or BSA for $5 \mathrm{~h}$. Cells were washed with warm PBS and incubated in $\alpha$-MEM without (basal) or with $100 \mathrm{nmol} / 1$ insulin for $30 \mathrm{~min}$. The medium was removed and 2-deoxy-D- $\left[{ }^{14} \mathrm{C}\right]$ glucose uptake $(18.5 \mathrm{kBq} / \mathrm{ml}, 10 \mu \mathrm{mol} / 1$ cold 2-DG) was added. The assay was stopped after $20 \mathrm{~min}$ by washing with ice-cold PBS three times. Cells were lysed in $30 \mathrm{mmol} / \mathrm{l} \mathrm{NaOH}$ and an aliquot was used for protein determination by the BCA method. Radioactivity was determined by liquid scintillation counting.

\section{Statistical analysis}

Statistical analysis was performed using unpaired Student's $t$ test. Two-way ANOVA with repeated measures was 
applied where appropriate and Student-Newman-Keuls post hoc analysis was performed. Statistical significance was set a priori at $p<0.05$.

\section{Results}

SCD1 expression is upregulated in the skeletal muscle of obese rodents

Previous studies in liver indicate that SCD1 expression is increased in obese and/or insulin resistant animals [2]. We first determined the effects of high-fat feeding on SCD1 expression in skeletal muscle. SCD1 protein expression was elevated by $\sim 35 \%$ in skeletal muscle of mice (C57Bl/6J) and rats (Wistar) made obese by high-fat diets (Fig. 1a). SCD1 content in the skeletal muscle of another rodent model of obesity/insulin resistance, the $d b / d b$ mouse, was increased compared with expression from muscle extracts of $d b /+$ mice (Fig. 1a). We next tested the efficacy of our experimental in vitro skeletal muscle system. SCD1 protein content in control siRNA-treated cells was not different from that in native L6 myotubes, whereas SCD1 siRNA reduced SCD1 protein by $40 \%$ (Fig. 1b). L6 myoblasts were also transfected with pcDNA3.1/Hygro-mSCD1 (L6 SCD1) or empty vector (L6) and rapidly differentiated into myotubes. SCD1 protein content in fully differentiated myotubes was increased 1.35-fold (Fig. 1c). Interestingly, the fold increase observed in L6 myotubes was similar to the increase seen in obese, insulin-resistant rodents compared with lean littermates (Fig. 1a).

SCD1 knockdown affects fatty acid metabolism in L6 myotubes

There were no differences between native L6 and control siRNA-treated cells for SCD1 protein or any metabolic parameter reported here (results not shown). Fatty acid oxidation (Fig. 2a) and fatty acid incorporation into triacylglycerol (Fig. 2b) were not different between treatments, whereas SCD1 knockdown resulted in a twofold increase in palmitate incorporation into DAG (Fig. 2c). We next examined the cellular contents of DAG and ceramide, fatty acid metabolites known to interfere directly with insulin signal transduction [3-5]. When cells were grown in fatty acid-free medium, SCD1 siRNA resulted in a two and threefold increase in cellular ceramide and DAG respectively, compared with control siRNA (Fig. 2d,e). When cells were exposed to exogenous palmitate, ceramide and DAG contents were increased above basal levels in both control and SCD1 siRNA cells. Ceramide content remained higher in SCD1 siRNA (Fig. 2d), whereas DAG content was not different between treatments (Fig. 2e).
SCD1 knockdown induces insulin resistance in L6 myotubes

Basal 2-deoxyglucose (2DG) uptake was not different between control and SCD1 siRNA-treated cells (Fig. 3a). Insulin-stimulated 2DG uptake was increased by about $70 \%$ above basal in control siRNA L6 myotubes, which is consistent with previous values reported for this cell line [6]. SCD1 siRNA abrogated the insulin-stimulated 2DG uptake observed in control cells (Fig. 3a), which coincided with ceramide and DAG accumulation (Fig. 2e,f). Treating L6 myotubes with $0.5 \mathrm{mmol} / 1$ palmitate for $5 \mathrm{~h}$ blunted insulin-stimulated 2DG uptake in control cells and 2DG uptake remained at basal rates in SCD1 siRNA cells. There were no differences in IRS1 Y785 phosphorylation or PI3 kinase p85 subunit association with IRS1 (results not shown). These data indicate that the decrements in $2 \mathrm{DG}$ uptake with SCD1 siRNA are not due to defective signalling at IRS1. Akt activity was increased by insulin and was partially blunted by palmitate treatment in both groups (Fig. 3b). Insulin-stimulated Akt phosphorylation on Ser473 was blunted by palmitate in control and SCD1 siRNA myotubes, which is consistent with the Akt activity measures. Akt phosphorylation was lower in L6 myotubes treated with SCD1 siRNA than in those treated with control siRNA, both without and with palmitate treatment. Collectively, these data demonstrate that SCD1 is essential for the maintenance of normal insulin-stimulated glucose transport in L6 myotubes and the defect in signalling appears to occur at the level of Akt.

SCD1 overexpression attenuates lipid metabolite accumulation in L6 myotubes

Our initial experiments demonstrated that SCD1 protected L6 myotubes from insulin resistance by preventing the accumulation of lipid metabolites; however, the addition of palmitate resulted in insulin resistance. To investigate whether SCD1 protected myotubes from palmitate-induced insulin resistance, L6 myoblasts were transfected with pcDNA3.1/ Hygro-mSCD1 (L6 SCD1) or empty vector (L6) and rapidly differentiated into myotubes. SCD1 overexpression resulted in a small $(12 \pm 1 \%)$ yet significant decrease in oxidation (Fig. 4a). This was not associated with changes in $5^{\prime}$-AMPactivated protein kinase expression or phosphorylation as determined by western blot analysis (results not shown). SCD1 overexpression reduced the incorporation of palmitate into diacylglycerol by $50 \%$, whereas label recovery in triacylglycerol was $40 \%$ greater (Fig. 4b,c). There was no difference in ceramide and DAG contents with SCD1 overexpression when cells were incubated in fatty acid-free medium (Fig. 4d,e). Consistent with the $\left[{ }^{3} \mathrm{H}\right]$ palmitate incorporation experiments, ceramide and DAG content were 
Fig. 1 Expression of SCD1 in skeletal muscle of lean and obese rodents and in L6 myotubes in vitro. (a) SCD1 protein expression in animals fed chow or high-fat diet and in lean $(+/-)$ or obese $(+/+) d b / d b$ mice $(n=4)$. Representative immunoblots are above the respective condition. *Different from lean. For $d b / d b$ in the upper panel, the samples were run on the same gel. The immunoblot was then cut and two different portions of the same gel are shown side by side. We deliberately show this as a 'split' figure. (b) SCD1 protein levels in native L6 myotubes, or L6 myotubes transfected with control (Con) or SCD1 siRNA. Cells were transfected with siRNA for 16-20 h and differentiated for 3 days, after which cells were harvested. Expression is presented as arbitrary units and normalised to native L6 myotubes (mean \pm SEM, $n=6$ ). $\alpha$-Actin was used as a loading control. *Different from L6 and control siRNA, $p<0.05$. (c) SCD1 protein content in L6 myoblasts that were transfected with pcDNA3.1/ Hygro-mSCD1 or an empty vector for $20 \mathrm{~h}$ then differentiated for 3 days (mean \pm SEM, $n=6) . \alpha$-Actin was used as a loading control. $* p<0.05$ vs L6 as determined by unpaired $t$ test

\section{a}
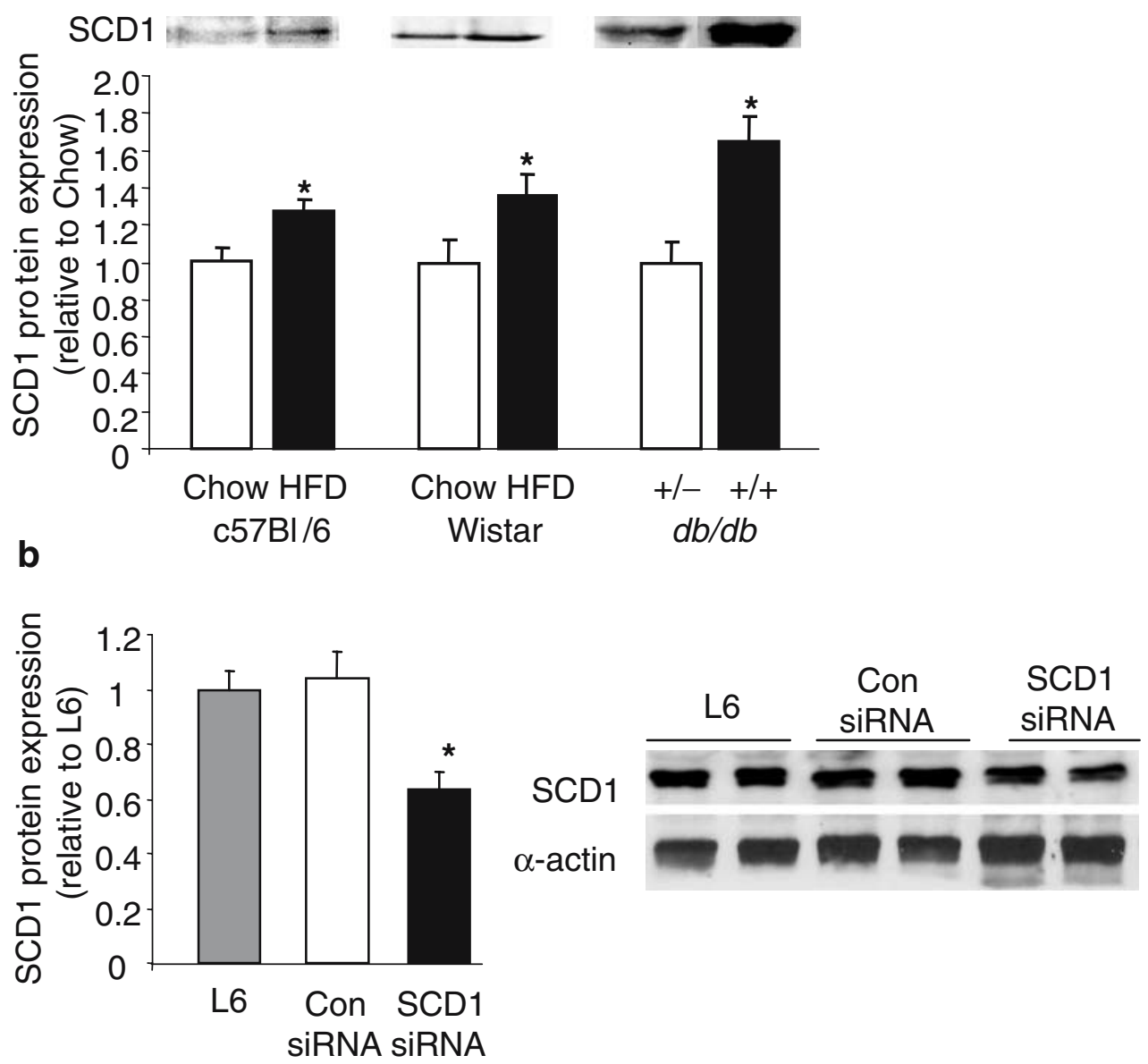

C

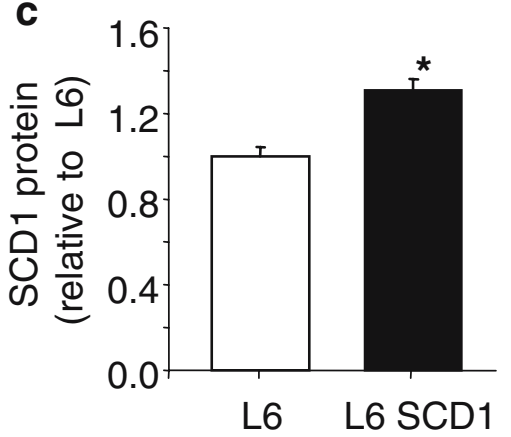

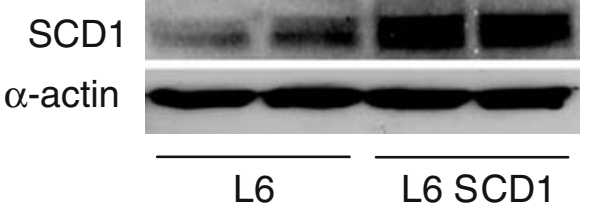

elevated fourfold in control cells incubated in palmitatecontaining media, whereas SCD1 overexpression attenuated this increase by about 50\%. Thus, despite small decreases in fatty acid oxidation, SCD1 overexpression results in partitioning of fatty acids into neutral lipid pools and limits the flux of palmitate into ceramides and DAG.

SCD1 overexpression protects L6 myotubes from fatty acid-induced insulin resistance

Uptake of 2DG in L6 SCD1 myotubes was increased both basally $(20 \%)$ and following insulin stimulation (14\%) compared with control L6 myotubes (Fig. 5a). The net increase in $2 \mathrm{DG}$ uptake in response to insulin was not different between cells. To examine whether SCD1 protects myotubes from insulin resistance in the presence of excess saturated fatty acids, cells were incubated for $5 \mathrm{~h}$ with $500 \mu \mathrm{mol} / 1$ palmitate. While about $75 \%$ of the insulinstimulated glucose uptake was lost in L6 myotubes pretreated with palmitate, only $35 \%$ of the insulin-stimulated glucose uptake was lost in L6 SCD1. IRS1 Y785 phosphorylation and PI3 kinase p85 subunit association were not different (results not shown). Conversely, palmitate decreased insulin-stimulated Akt Ser473 phosphoryla- 


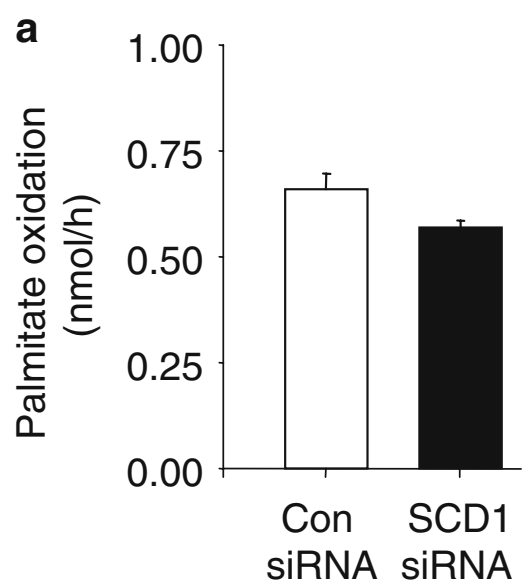

d

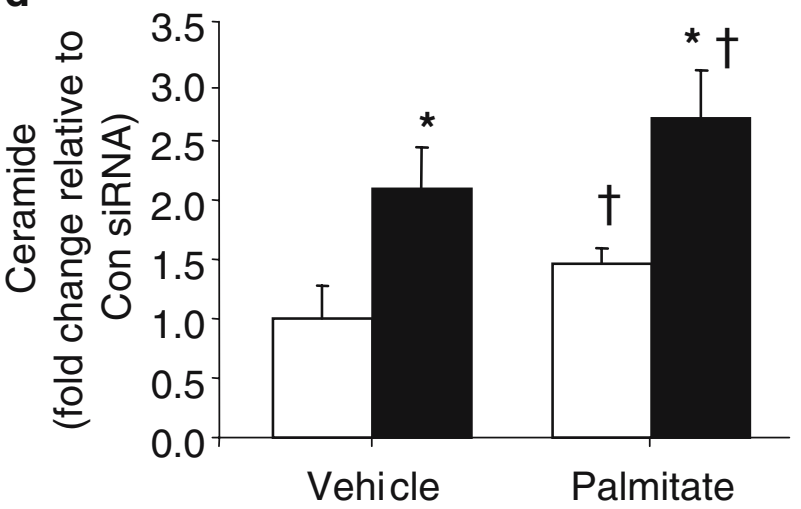

Fig. 2 SCD1 siRNA attenuates fatty acid oxidation and increases the incorporation of fatty acids into diacylglycerol and ceramide. L6 myotubes were transfected with control (Con) siRNA (open bars) or SCD1 siRNA (closed bars), differentiated for 3 days and assessed for fatty acid metabolism using $0.5 \mathrm{mmol} / 1\left[{ }^{3} \mathrm{H}\right]$ palmitate conjugated with $2 \%$ fatty acid-free bovine serum albumin. Results are expressed as $\mathrm{nmol} / \mathrm{h}$ palmitate oxidation (a), palmitate incorporation into triacylglycerol (TAG) (b) or incorporation into diacylglycerol (c),

tion to a greater extent in L6 (35\%) compared with L6 SCD1 cells $(20 \%)$ (Fig. 5c). There was no difference in total Akt protein. Taken together, these results indicate that SCD1 partially protects skeletal muscle cells from fatinduced insulin resistance in vitro, which appears to be mediated at the level of Akt.

\section{Discussion}

In this study, we sought to clarify the role of SCD1 in the regulation of fatty acid metabolism and insulin action in skeletal muscle cells. Previous studies have presented contrasting data with respect to SCD1 and cell function, some indicating that SCD1 prevents lipotoxicity and others suggesting that SCD1 deficiency reduces the accumulation of muscle lipids and provides resistance against diet-

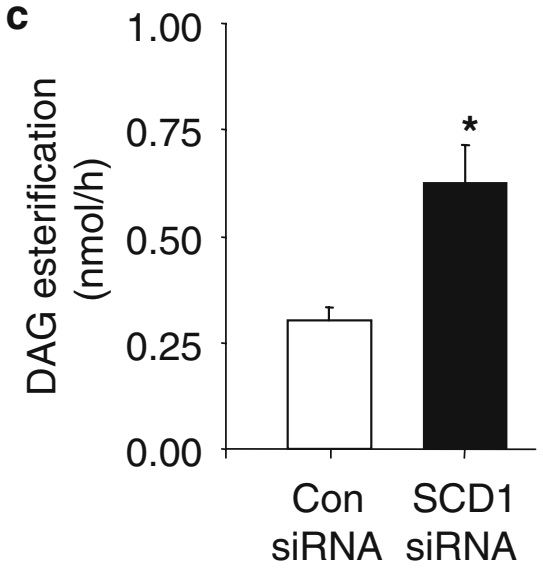

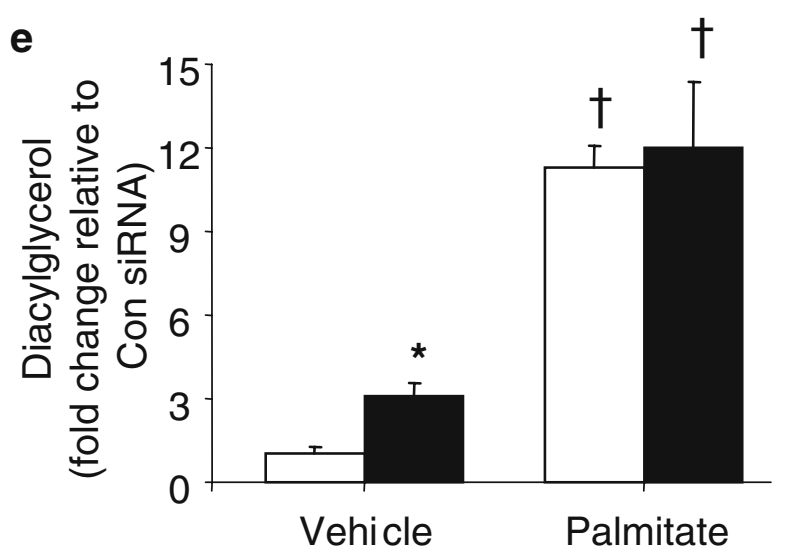

and represent mean \pm SEM ( $n=10$ individual wells). ${ }^{*} p<0.05$ as assessed by unpaired $t$ test. Ceramide (d) and DAG (e) contents were assessed in myotube lysates after $5 \mathrm{~h}$ incubation with BSA alone or $0.5 \mathrm{mmol} / 1$ palmitate conjugated to $2 \%$ fatty acid-free BSA. Results are expressed as fold change relative to control siRNA vehicle and represent mean \pm SEM ( $n=10$ individual wells). ${ }^{*} p<0.05$ vs vehicle, $\dagger p<0.05$ vs vehicle within the same group

induced obesity. Reducing SCD1 expression in L6 myotubes increased ceramide and DAG accumulation and promoted insulin resistance. In contrast, increasing SCD1 provided a protective effect against fatty acid-induced insulin resistance by limiting lipid metabolite accumulation and partially ameliorating Akt inhibition. These data demonstrate, therefore, that SCD1 prevents fatty acidinduced ceramide and DAG accumulation and insulin resistance in skeletal muscle myotubes in vitro.

Studies of mice with whole-body genetic ablation of SCD1 [21, 24] and of mice treated with SCD1 antisense oligonucleotide inhibitors [25] have implicated SCD1 as a cause of obesity and insulin resistance. SCD1-/- mice are leaner than wild-type controls and are hyperphagic, yet accumulate less adipose tissue and are resistant to dietinduced weight gain $[21,24,25]$. These changes are associated with numerous perturbations in metabolic func- 


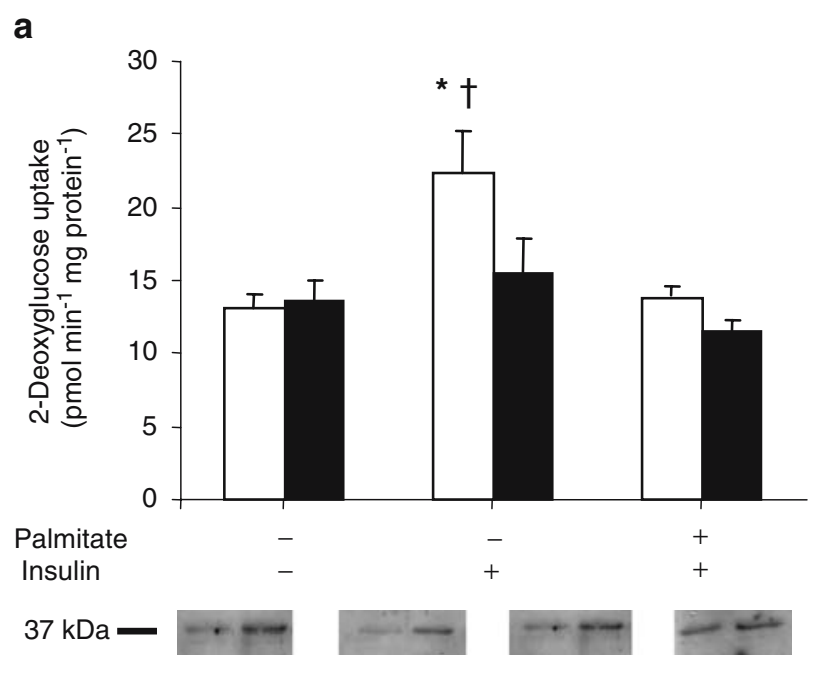

b

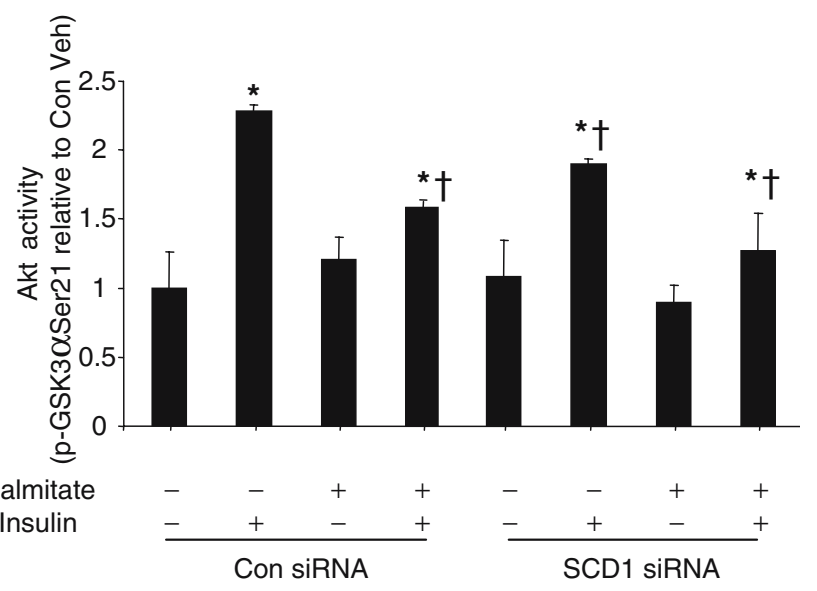

C
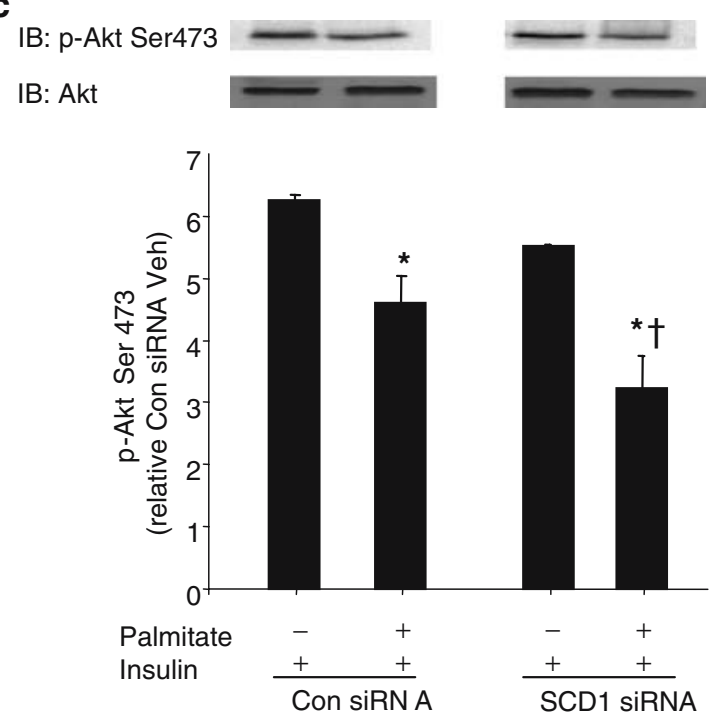

4 Fig. 3 Glucose uptake and Akt activity are attenuated by SCD1 siRNA. (a) Uptake of 2-deoxyglucose was assessed in control (Con) siRNA (open bars) and SCD1 siRNA (closed bars) myotubes following $5 \mathrm{~h}$ treatment with $2 \%$ fatty acid-free BSA or $0.5 \mathrm{mmol} / 1$ palmitate conjugated to $2 \%$ fatty acid-free BSA, with or without $100 \mathrm{nmol} / 1$ insulin. (b) In similar experiments, myotubes were treated as described above and were lysed for subsequent immunoprecipitation with anti-Akt for assessment of Akt activity. Bars represent mean \pm SEM $(n=4$ individual wells). ${ }^{*} p<0.05$ vs vehicle within the same group, $\dagger p<0.05$ vs control siRNA palmitate. (c) Cell lysates were also probed for phospho-Akt Ser 473 and total Akt protein content. Representative immunoblots are shown above each condition. ${ }^{*} p<0.05$ vs insulin of the same group, $\dagger p<0.05$ vs control siRNA palmitate + insulin. All bars represent mean \pm SEM ( $n=4-12$ individual wells)

tion including AMP-activated protein kinase (AMPK) activation and greater fatty acid oxidation [33, 34], reduced hepatic de novo fatty acid synthesis [25] and improved insulin signalling $[24,35,36]$. These mice also exhibit an increased metabolic rate, upregulation of genes associated with lipid oxidation and suppression of lipogenic genes [24, 25]. Therefore, whilst the clinical outcomes of global SCD1 ablation appear to be largely desirable, aside from the development of alopecia, the compensatory metabolic perturbations in these whole-animal models make it difficult to ascertain the direct function of SCD1 in specific tissues, which provided the impetus for undertaking the present series of studies.

It should also be noted that there are many direct actions of SCD1 per se that would argue against this enzyme being implicated in directly causing insulin resistance. First, the primary role of SCD1 is to convert saturated fatty acids (primarily 16:0, 18:0) to monounsaturated fatty acids (16:1, 18:1) [16, 37], which are less likely to interfere with insulin signal transduction. Second, incubating cells with saturated fatty acids leads to insulin resistance, subsequent to increases in ceramide and DAG content [11-13]. Third, global SCD1 ablation reduces skeletal muscle ceramide accumulation [33] and improves insulin signalling and glucose tolerance [35]. Altering SCD1 protein content in L6 myotubes induced modest changes in fatty acid oxidation (i.e. $<20 \%$ ), changes that are unlikely to underpin the observed changes in lipid metabolites discussed here (i.e. 100-300\%). Ceramide and DAG were markedly elevated in SCD1-deficient cells, even when excess palmitate was not present in the culture medium, suggesting a critical protective role of SCD1 in normal lipid metabolism. Increasing SCD1 content largely prevented the palmitate-induced increase in ceramide and DAG.

These data are in contrast with observations in SCD1deficient mice showing reduced ceramide in oxidative muscles [33]. In SCD1-deficient mice, both serine palmitoyl transferase, a key enzyme in ceramide synthesis, and fatty acyl CoA, a substrate for de novo synthesis, were 

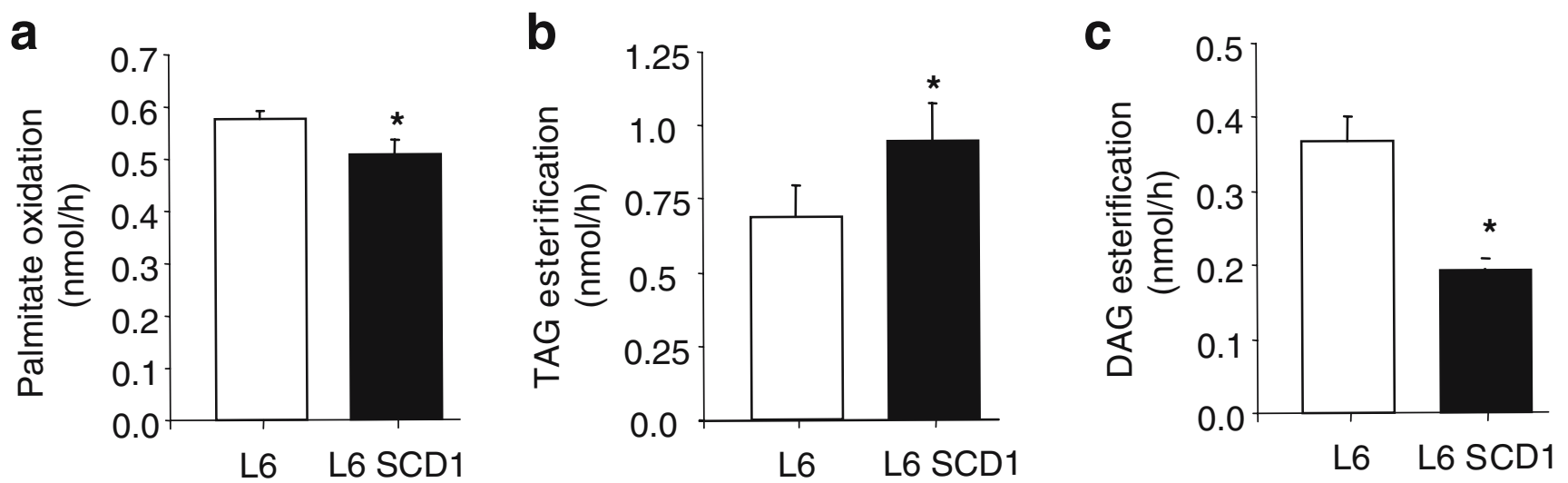

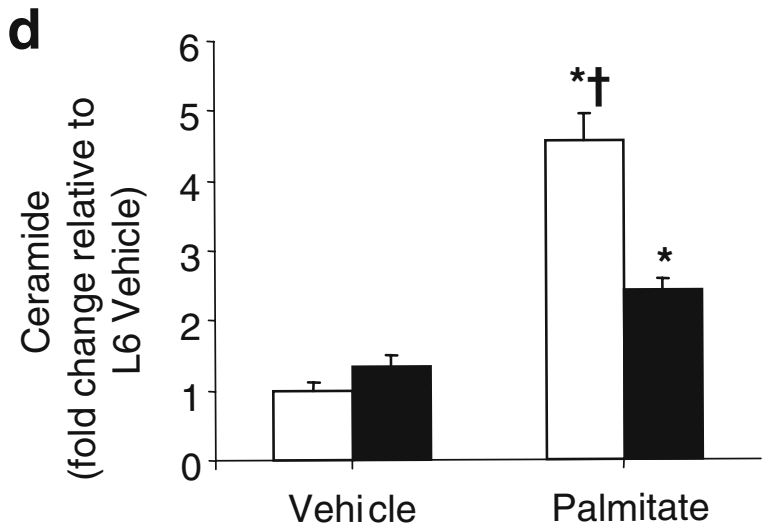

Fig. 4 Transient SCD1 overexpression in L6 myotubes attenuates diacylglycerol and ceramide accumulation but results in triacylglycerol accumulation. L6 myoblasts were transfected with SCD1 (closed bars) or an empty vector (open bars) for $20 \mathrm{~h}$ and cells were differentiated for 3 days. Fatty acid metabolism was assessed using $0.5 \mathrm{mmol} / \mathrm{l}$ $\left[{ }^{3} \mathrm{H}\right]$ palmitate conjugated to $2 \%$ fatty acid-free BSA. Results are expressed as $\mathrm{nmol} / \mathrm{h}$ palmitate oxidation (a), palmitate incorporation

reduced. Moreover, fatty acid oxidation was increased in SCD1 $-/-$ mice, making it difficult to establish the precise role of SCD1 in ceramide synthesis. In SCD1 siRNA muscle cells, the increased ceramide levels could be explained by increased substrate provision, given that fatty acid uptake was marginally increased (1.2-fold) and oxidation remained unchanged. However, the disproportionate increase in ceramide (and DAG) content (two- to threefold), whose pools are principally increased by saturated fatty acids, suggest that SCD1 limits excess accumulation of bioactive lipid species. Indeed, when SCD1 was overexpressed, about $50 \%$ of the palmitateinduced increase in ceramide (and DAG) was prevented despite a small (1.12-fold) decrease in palmitate oxidation. Thus, the present data indicate that, at least in vitro: (1) SCD1 is required to prevent the accumulation of ceramide and DAG in skeletal muscle under basal conditions; and (2) SCD1 is essential to minimise palmitate-induced increases in ceramide and DAG.

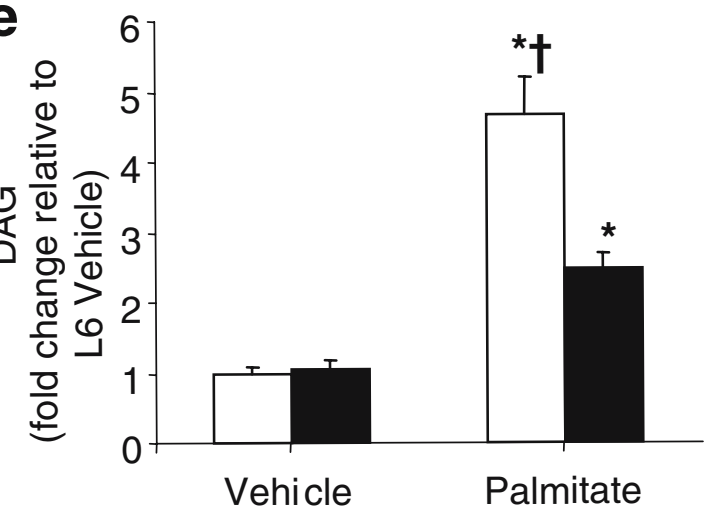

into triacylglycerol (b) or incorporation into diacylglycerol (c), and represent mean SEM ( $n=10$ individual wells). ${ }^{*} p<0.05$ as assessed by unpaired $t$ test. Ceramide (d) and DAG (e) contents were assessed in myotube lysates after $5 \mathrm{~h}$ incubation with BSA alone or $0.5 \mathrm{mmol} / \mathrm{l}$ palmitate conjugated to $2 \%$ fatty acid-free BSA. Results are expressed as fold change relative to L6 vehicle and represent mean SEM ( $n=6$ individual wells). ${ }^{*} p<0.05$ vs vehicle, ${ }^{\dagger} p<0.05$ vs L6

Although our findings of a protective role of SCD1 are consistent with data on other cell types [17, 18, 38], some authors [29] have suggested that elevated skeletal muscle SCD1 expression contributes to abnormal lipid metabolism and the aetiology of obesity. In this previous study [29], SCD1 overexpression decreased fatty acid oxidation and increased triacylglycerol synthesis. While our data largely support these observations, triacylglycerol accumulation per se is unlikely to cause insulin resistance [39] or lipotoxicity $[17,18,38]$, and in fact may provide protection by providing a reservoir for the deposition of other fatty acyl-CoA metabolites. Importantly, our data add to these previous observations and indicate that triacylglycerol accumulation in SCD1-overexpressing cells protects cells from DAG and ceramide accumulation. Thus, the induction of SCD1 in skeletal muscle of obese, insulin-resistant phenotypes may be a consequence rather than a cause of insulin resistance and may be initiated to prevent the progression of insulin resistance. 
In the present study, insulin-stimulated 2DG uptake was abrogated in SCD1 siRNA cells and endogenous levels of SCD1 did not prevent palmitate-induced insulin resistance. In contrast, overexpressing SCD1 prevented most of the palmitate-induced reductions in insulin-stimulated glucose uptake, possibly by inhibition of ceramide accumulation. Ceramides negatively regulate insulin action downstream of phosphatidylinositol 3-kinase by protein phosphatase $2 \mathrm{~A}$ and protein kinase $\mathrm{C} \zeta$-mediated dephosphorylation of Akt and preventing Akt plasma membrane recruitment [40-43]. The finding of reduced Akt phosphorylation and activity indicates that ceramide accumulation contributes to the

a

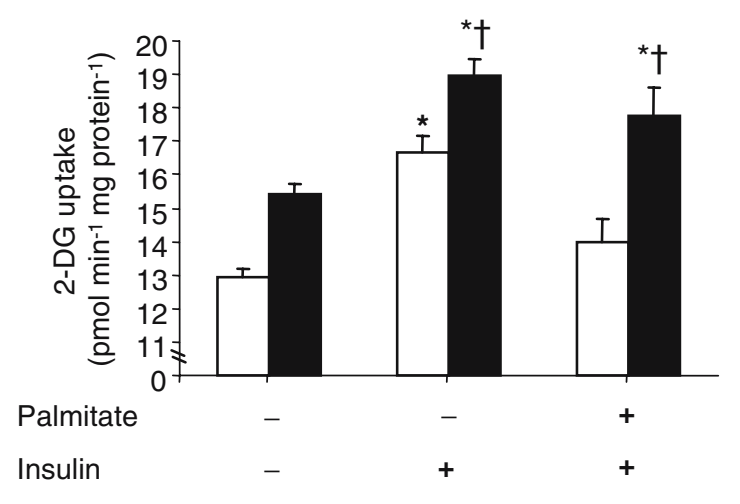

b

IB: p-Akt Ser4 73

IB: Akt

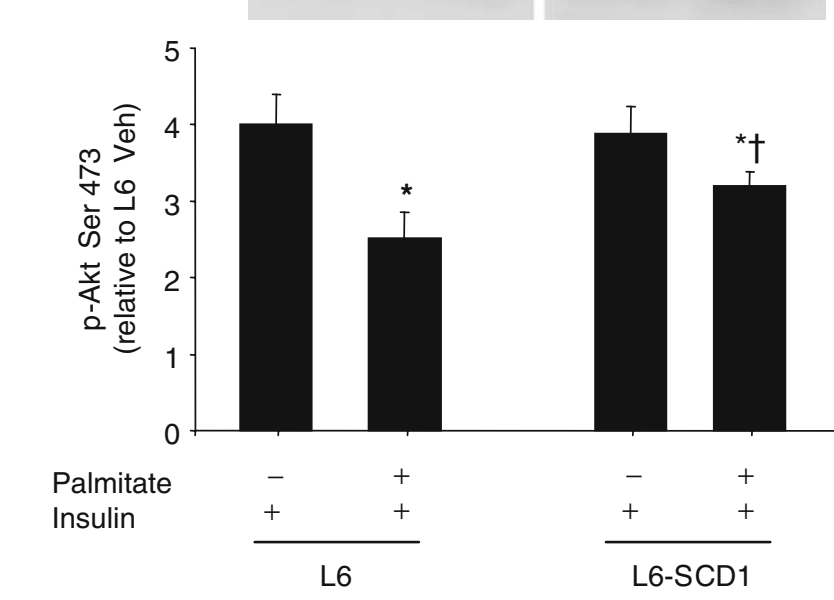

Fig. 5 Glucose uptake and insulin signalling with SCD1 overexpression. (a) 2-Deoxyglucose $(2-D G)$ uptake was assessed in myotubes following $5 \mathrm{~h}$ treatment with $2 \%$ fatty acid-free BSA or $0.5 \mathrm{mmol} / 1$ palmitate conjugated to $2 \%$ fatty acid-free BSA, with or without $100 \mathrm{nmol} / 1$ insulin. Myotubes were transfected with SCD1 (closed bars) or an empty vector (open bars). (b) In similar experiments, myotubes were treated as described above and were lysed for subsequent determination of phospho-Akt Ser 473 and total Akt protein expression. Representative immunoblots are shown above each condition. ${ }^{*} p<0.05$ vs vehicle within the same group, ${ }^{\dagger} p<0.05$ vs L6 $(n=8)$ palmitate-mediated suppression of glucose uptake in L6 myotubes. It is unlikely that SCD1 content alters proximal components of the insulin signalling cascade, given that tyrosine phosphorylation of IRS1 and p85 association with IRS1 were not different between treatments. Thus, elevated ceramide synthesis and suppressed Akt action appear to contribute to the palmitate-induced insulin resistance.

The role of SCD1 in insulin signalling in vivo remains unresolved. SCD1 is elevated in tissues of insulin-resistant mice (Fig. 1) and SCD1-/- mice exhibit improved glucose tolerance and enhanced insulin signalling [24, 35, 36]. Whether SCD1 is coupled to the insulin signalling pathway cannot be determined because decreased adiposity may have contributed to the improved insulin signalling, as weight loss per se may lead to enhanced insulin signalling either directly or via secretion of adipokines such as TNF- $\alpha$ and resistin, which are implicated in insulin resistance [for review see 44]. SCD1-deficient aP2-nSREBP-1c mice do not exhibit greater insulin sensitivity than control aP2nSREBP-1c mice, despite reduced liver steatosis [22], suggesting no direct role of SCD1 in insulin resistance. Moreover, SCD1 mRNA/activity is increased after treatment with the glucose-lowering drug rosiglitazone, indicating that SCD1 may play a role in insulin sensitisation [45, 46]. The present studies indicate that the induction of SCD1 in obese, insulin-resistant phenotypes may be a consequence rather than a cause of insulin resistance and may be initiated to prevent further insulin resistance. Whilst we were completing our studies, another group reported that SCD1 overexpression induced insulin resistance in L6 myotubes [47]. However, the apparent insulin resistance observed in these cells appeared to be a function of reduced global glucose uptake (i.e. about threefold reduction in basal glucose uptake in L6-SCD1 vs L6 native). The L6SCD1 cells exhibited an identical percentage increase in glucose uptake with insulin stimulation, and whereas insulin-stimulated glucose uptake was reduced in native L6 cells exposed to palmitate, glucose uptake was unaffected in L6-SCD1 cells after palmitate treatment.

The data from the present study do not shed light on the role of SCD1 in the aetiology of whole-body insulin sensitivity. They do, however, indicate that SCD1 protects muscle cells from fatty acid-induced insulin resistance by reducing ceramide and DAG accumulation. This interpretation supports studies in beta cells, lung fibroblasts and Chinese hamster ovary cells $[17,18,38]$ that describe a role for SCD1 in protection against the toxic effects of palmitate and support the model of cellular lipid metabolism, in which triacylglycerol accumulation protects against lipotoxicity.

Acknowledgements M. J. Watt is supported by a Peter Doherty Postdoctoral Fellowship and M. A. Febbraio by a Senior Research Fellowship from the National Health and Medical Research Council of 
Australia. R. J. Southgate is supported by an Australian Postgraduate Award. This study was funded by grants from the Australian Research Council (DP0450436) and the Royal Melbourne Institute of Technology.

\section{References}

1. Eckel RH, Grundy SM, Zimmet PZ (2005) The metabolic syndrome. Lancet 365:1415-1428

2. Krssak M, Falk PK, Dresner A et al (1999) Intramyocellular lipid concentrations are correlated with insulin sensitivity in humans: a 1H NMR spectroscopy study. Diabetologia 42:113-116

3. Pan DA, Lillioja S, Kriketos AD et al (1997) Skeletal muscle triglyceride levels are inversely related to insulin action. Diabetes 46:983-988

4. Storlien LH, Jenkins AB, Chisholm DJ, Pascoe WS, Khouri S, Kraegen EW (1991) Influence of dietary fat composition on development of insulin resistance in rats. Relationship to muscle triglyceride and omega-3 fatty acids in muscle phospholipid. Diabetes 40:280-289

5. Oakes ND, Cooney GJ, Camilleri S, Chisholm DJ, Kraegen EW (1997) Mechanisms of liver and muscle insulin resistance induced by chronic high-fat feeding. Diabetes 46:1768-1774

6. Jucker BM, Cline GW, Barucci N, Shulman GI (1999) Differential effects of safflower oil versus fish oil feeding on insulinstimulated glycogen synthesis, glycolysis, and pyruvate dehydrogenase flux in skeletal muscle: a $13 \mathrm{C}$ nuclear magnetic resonance study. Diabetes 48:134-140

7. Itani SI, Ruderman NB, Schmieder F, Boden G (2002) Lipidinduced insulin resistance in human muscle is associated with changes in diacylglycerol, protein kinase C, and IkappaB-alpha. Diabetes 51:2005-2011

8. Boden G, Chen X (1995) Effects of fat on glucose uptake and utilization in patients with non-insulin-dependent diabetes. J Clin Invest 96:1261-1268

9. Boden G, Lebed B, Schatz M, Homko C, Lemieux S (2001) Effects of acute changes of plasma free fatty acids on intramyocellular fat content and insulin resistance in healthy subjects. Diabetes 50:1612-1617

10. Yu C, Chen Y, Cline GW et al (2002) Mechanism by which fatty acids inhibit insulin activation of insulin receptor substrate-1 (IRS-1)-associated phosphatidylinositol 3-kinase activity in muscle. J Biol Chem 277:50230-50236

11. Schmitz-Peiffer C, Craig DL, Biden TJ (1999) Ceramide generation is sufficient to account for the inhibition of the insulin-stimulated PKB pathway in $\mathrm{C} 2 \mathrm{C} 12$ skeletal muscle cells pretreated with palmitate. J Biol Chem 274:24202-24210

12. Montell E, Turini M, Marotta M et al (2001) DAG accumulation from saturated fatty acids desensitizes insulin stimulation of glucose uptake in muscle cells. Am J Physiol Endocrinol Metab 280:E229-E237

13. Chavez JA, Knotts TA, Wang LP et al (2003) A role for ceramide, but not diacylglycerol, in the antagonism of insulin signal transduction by saturated fatty acids. J Biol Chem 278:10297-10303

14. Sinha S, Perdomo G, Brown NF, O’Doherty RM (2004) Fatty acid-induced insulin resistance in L6 myotubes is prevented by inhibition of activation and nuclear localization of nuclear factor kappa B. J Biol Chem 279:41294-41301

15. Yuan M, Konstantopoulos N, Lee J et al (2001) Reversal of obesity- and diet-induced insulin resistance with salicylates or targeted disruption of Ikkbeta. Science 293:1673-1677

16. Ntambi JM, Miyazaki M (2004) Regulation of stearoyl-CoA desaturases and role in metabolism. Prog Lipid Res 43:91-104
17. Scaglia N, Igal RA (2005) Stearoyl-CoA desaturase is involved in the control of proliferation, anchorage-independent growth, and survival in human transformed cells. J Biol Chem 280: 25339-25349

18. Listenberger LL, Han X, Lewis SE et al (2003) Triglyceride accumulation protects against fatty acid-induced lipotoxicity. Proc Natl Acad Sci USA 100:3077-3082

19. Gaster M, Rustan AC, Beck-Nielsen H (2005) Differential utilization of saturated palmitate and unsaturated oleate: evidence from cultured myotubes. Diabetes 54:648-656

20. Storlien LH, Higgins JA, Thomas TC et al (2000) Diet composition and insulin action in animal models. Br J Nutr 83 (Suppl 1):S85-90

21. Cohen P, Miyazaki M, Socci ND et al (2002) Role for stearoylCoA desaturase-1 in leptin-mediated weight loss. Science 297: 240-243

22. Asilmaz E, Cohen P, Miyazaki M et al (2004) Site and mechanism of leptin action in a rodent form of congenital lipodystrophy. $\mathrm{J}$ Clin Invest 113:414-424

23. Biddinger SB, Almind K, Miyazaki M, Kokkotou E, Ntambi JM, Kahn CR (2005) Effects of diet and genetic background on sterol regulatory element-binding protein-1c, stearoyl-CoA desaturase 1 , and the development of the metabolic syndrome. Diabetes 54: $1314-1323$

24. Ntambi JM, Miyazaki M, Stoehr JP et al (2002) Loss of stearoylCoA desaturase-1 function protects mice against adiposity. Proc Natl Acad Sci USA 99:11482-11486

25. Jiang G, Li Z, Liu F, Ellsworth K et al (2005) Prevention of obesity in mice by antisense oligonucleotide inhibitors of stearoylCoA desaturase-1. J Clin Invest 115:1030-1038

26. Lee SH, Dobrzyn A, Dobrzyn P, Rahman SM, Miyazaki M, Ntambi JM (2004) Lack of stearoyl-CoA desaturase 1 upregulates basal thermogenesis but causes hypothermia in a cold environment. J Lipid Res 45:1674-1682

27. van Hall G, Sacchetti M, Radegran G, Saltin B (2002) Human skeletal muscle fatty acid and glycerol metabolism during rest, exercise and recovery. J Physiol 543:1047-1058

28. Baron AD, Brechtel G, Wallace P, Edelman SV (1988) Rates and tissue sites of non-insulin- and insulin-mediated glucose uptake in humans. Am J Physiol 255:E769-E774

29. Hulver MW, Berggren JR, Carper MJ et al (2005) Elevated stearoyl-CoA desaturase-1 expression in skeletal muscle contributes to abnormal fatty acid partitioning in obese humans. Cell Metab 2:251-261

30. Goodpaster BH, He J, Watkins S, Kelley DE (2001) Skeletal muscle lipid content and insulin resistance: evidence for a paradox in endurance-trained athletes. J Clin Endocrinol Metab 86:57555761

31. Watt MJ, Steinberg GR, Heigenhauser GJ, Spriet LL, Dyck DJ (2003) Hormone-sensitive lipase activity and triacylglycerol hydrolysis are decreased in rat soleus muscle by cyclopiazonic acid. Am J Physiol Endocrinol Metab 285:E412-E419

32. Preiss J, Loomis CR, Bishop WR, Stein R, Niedel JE, Bell RM (1986) Quantitative measurement of sn-1,2-diacylglycerols present in platelets, hepatocytes, and ras- and sis-transformed normal rat kidney cells. J Biol Chem 261:8597-8600

33. Dobrzyn A, Dobrzyn P, Lee SH et al (2005) Stearoyl-CoA desaturase-1 deficiency reduces ceramide synthesis by downregulating serine palmitoyltransferase and increasing beta-oxidation in skeletal muscle. Am J Physiol Endocrinol Metab 288: E599-E607

34. Dobrzyn P, Dobrzyn A, Miyazaki M et al (2004) Stearoyl-CoA desaturase 1 deficiency increases fatty acid oxidation by activating AMP-activated protein kinase in liver. Proc Natl Acad Sci USA 101:6409-6414 
35. Rahman SM, Dobrzyn A, Dobrzyn P et al (2003) Stearoyl-CoA desaturase 1 deficiency elevates insulin-signaling components and down-regulates protein-tyrosine phosphatase $1 \mathrm{~B}$ in muscle. Proc Natl Acad Sci USA 100:11110-11115

36. Rahman SM, Dobrzyn A, Lee SH, Dobrzyn P, Miyazaki M, Ntambi JM (2005) Stearoyl-CoA desaturase 1 deficiency increases insulin signaling and glycogen accumulation in brown adipose tissue. Am J Physiol Endocrinol Metab 288:E381-E387

37. Enoch HG, Catala A, Strittmatter P (1976) Mechanism of rat liver microsomal stearyl-CoA desaturase. Studies of the substrate specificity, enzyme-substrate interactions, and the function of lipid. J Biol Chem 251:5095-5103

38. Busch AK, Gurisik E, Cordery DV et al (2005) Increased fatty acid desaturation and enhanced expression of stearoyl coenzyme A desaturase protects pancreatic $\beta$-cells from lipoapoptosis. Diabetes 54:2917-2924

39. Kelley DE, Goodpaster BH, Storlien L (2002) Muscle triglyceride and insulin resistance. Annu Rev Nutr 22:325-346

40. Stratford S, Hoehn KL, Liu F, Summers SA (2004) Regulation of insulin action by ceramide: dual mechanisms linking ceramide accumulation to the inhibition of Akt/protein kinase B. J Biol Chem 279:36608-36615

41. Hajduch E, Balendran A, Batty IH et al (2001) Ceramide impairs the insulin-dependent membrane recruitment of protein kinase $B$ leading to a loss in downstream signalling in L6 skeletal muscle cells. Diabetologia 44:173-183

42. Powell DJ, Turban S, Gray A, Hajduch E, Hundal HS (2004) Intracellular ceramide synthesis and protein kinase Czeta activation play an essential role in palmitate-induced insulin resistance in rat L6 skeletal muscle cells. Biochem J 382:619-629

43. Powell DJ, Hajduch E, Kular G, Hundal HS (2003) Ceramide disables 3-phosphoinositide binding to the pleckstrin homology domain of protein kinase B (PKB)/Akt by a PKCzeta-dependent mechanism. Mol Cell Biol 23:7794-7808

44. Lazar MA (2005) How obesity causes diabetes: not a tall tale. Science 307:373-375

45. Singh Ahuja H, Liu S, Crombie DL et al (2001) Differential effects of rexinoids and thiazolidinediones on metabolic gene expression in diabetic rodents. Mol Pharmacol 59:765-773

46. Riserus U, Tan GD, Fielding BA et al (2005) Rosiglitazone increases indexes of stearoyl-CoA desaturase activity in humans: link to insulin sensitization and the role of dominant-negative mutation in peroxisome proliferator-activated receptor-gamma. Diabetes 54:1379-1384

47. Voss MD, Beha A, Tennagels N et al (2005) Gene expression profiling in skeletal muscle of Zucker diabetic fatty rats: implications for a role of stearoyl-CoA desaturase 1 in insulin resistance. Diabetologia 48:2622-2630 